\title{
Der Flug der Schmetterlinge
}

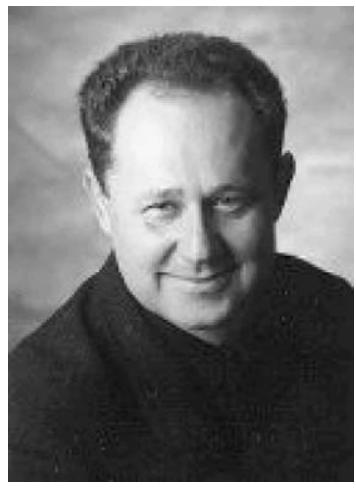

Prof. Dr. med. Manfred Wildner
Der Flug der Schmetterlinge bezaubert mit seiner Leichtigkeit und scheinbaren Spontaneität - dabei ist er nur auf den ersten Blick spielerisch-taumelig. Tatsächlich passen sich die Vertreter der Ordnung Lepidoptera zielgerichtet ihrer Umwelt an und nutzen dabei geschickt den Wind als wichtiges Transportmittel. Viele der bisher bekannten 180000 Arten leben in den Tropen. Dabei sind durchaus auch Höhenflüge möglich: In den Alpen etwa ist der Matterhornbär bis auf eine Höhe von $3200 \mathrm{~m}$ zu finden. Vielleicht war es diese bezaubernde Leichtigkeit und scheinbare Spontaneität, welche die altgriechische $\mathrm{Na}$ mensgebung für Schmetterlinge beeinflusst hat, nämlich mit „Psyche“ (Hauch, Atem Seele).

Nützlich sind die Spinnfäden der ostasiatischen Familie der Seidenspinner als Textilfasern, schädlich sind die Aktivitäten der Kleidermotten. Beängstigend wäre eine Eigenschaft, die in der Theorie komplexer, nicht linearer dynamischer Systeme als „Schmetterlingseffekt“ bezeichnet wird. Gemeint ist, dass geringfügige Änderungen in den Anfangsbedingungen von komplexen Systemen in der langfristigen Perspektive unerwartete Ergebnisse zeitigen können. Diese fehlende Vorhersagbarkeit wurde im Zusammenhang mit Atmosphärenmodellen bildhaft in dem Satz ausgedrückt: „Kann der Flügelschlag eines Schmetterlings in Brasilien einen Tornado in Texas auslösen?" $[1,2]$. In der Tat ein bedrohliches Szenario. Auch im Bereich der Gesundheitswissenschaften werden die Ebenen von Bevölkerungen und gesellschaftlichen Systemen und Subsystemen in ihren Reaktionen zunehmend als komplex wahrgenommen. Dabei kommen, anders als beim einfachen Flügelschlag eines Schmetterlings, auch „komplexe“ Multikomponenten- oder Mehrebenen-Interventionen zur Anwendung, welche in ihrer Kombination mehr sein können und sollen als die Summe ihrer Teile. Typische Anwendungsfelder finden sich in der Prävention und Gesundheitsförderung. Dies hat Auswirkungen auf die Evaluationsmethodik: Diskutiert werden der Einsatz von quantitativen und qualitativen Methoden in verschiedenen Evaluationsphasen, ihre Integration und ein zusätzlicher Fokus auf die Prozesse, neben der klassischen Ergebnisevaluation $[3,4]$. Anlass für derartige Multikomponenten-Interventionen geben schwer zu beeinflussende, gesellschaftlich hoch relevante Gesundheitsprobleme wie der Alkoholmissbrauch oder die globale Adipositas-Epidemie [5]. Die Weltgesundheitsorganisation hat in diesem Zusammenhang den aus der Sozialplanung stammenden Begriff der „wicked problems“ (hartnäckige Pro- bleme) übernommen: Gemeint sind schwer zu definierende Probleme, für welche es keine definitiven Lösungen gibt, sondern nur gute oder weniger gute Herangehensweisen [6].

Hartnäckige Probleme sind gleichzeitig auch Herausforderungen für hartnäckige Wissenschaftler. Dies hat zu interessanten Perspektivwechseln bei Evaluationen im Bereich von Public Health geführt. Stehen in der klassischen Therapie- und Risikoforschung die Reduktion und Kontrolle der Einflussgrößen durch kontrollierte Bedingungen und Adjustierungen im Vordergrund, so fokussiert die Wirkungsforschung bei Interventionen in komplexen Zusammenhängen - z.B. in Prävention und Gesundheitsförderung - gerade auf die Erfolg versprechenden oder Erfolg verhindernden Kontextfaktoren: eine Revolution der Perspektive.

Dies verbindet sich auch mit einem erweiterten Kausalverständnis: Der einfache Ansatz einer einzelnen effektiven Ursache wird zunehmend ersetzt durch eine Kombination von Ursachen und Ursachenkontexten. Eine effektive Ursache entsteht dabei erst in Kombination von multiplen Teilursachen bzw. Kontexten [7,8]. Ein so erweitertes Kausalverständnis liefert z. B. ein Denkmodell dafür, warum Lungenkrebs zwar in den allermeisten Fällen mit Tabakkonsum ursächlich verknüpft ist (Rauchen als notwendige Ursache), jedoch nicht bei allen Rauchern eine zwangsläufige Erkrankung darstellt (Rauchen ist für sich alleine keine hinreichende Ursache). Ähnlich könnten Zusammenhänge im Bereich der Adipositas-Epidemie gelagert sein. Sind wichtige Teilursachen eventuell in unseren Erbanlagen zu suchen? Die Wechselwirkungen zwischen äußeren Ursachen und inneren, genetisch fixierten Veranlagungen („Gen-Umwelt-Interaktionen“) können wiederum mit der alten aristotelischen Begrifflichkeit von äußeren Wirkursachen und inneren Formursachen verstanden werden. Kommt der Wunsch nach einem bestimmten Körperbild hinzu, welcher dann die Pfunde auf der Waage nach oben oder unten treibt, wäre auch die aristotelische Zielursache mit im Spiel [9]. Im Umgang mit Ursachen- und Wirkungsforschung in komplexen Systemen sei auch an das Ökonomieprinzip des spätscholastischen Philosophen Wilhelm von Ockham erinnert werden: „Entia non sunt multiplicanda sine necessitate“ (Die Dinge sollten nicht unnötig verkompliziert werden) [10]. Damit soll nicht einer unangemessenen Vereinfachung das Wort geredet werden, wohl aber einer der gegebenen Komplexität angemessenen theoretischen Betrachtung: Komplexität ist kein Selbstzweck 
und sollte nicht zum sicheren Fluchtpunkt in wissenschaftlichen Diskursen werden.

Was folgt daraus für die Wissenschaft und Praxis der Bevölkerungsgesundheit/Medizin? Sprachlich und gedanklich kann zwischen einfachen und kombinierten („komplexen“) Interventionen sowie einfachen und komplexen Problemlagen unterschieden werden - gesellschaftliche Systeme und Subsysteme als „Tatorte“ sind grundsätzlich komplex. Letztlich ist Gesundheit das Ergebnis einer Vielzahl von komplexen Funktionen und in Wechselwirkung stehenden Strukturen - eine Herausforderung auch für neue wissenschaftliche Zweige wie Komplexwissenschaften und Systembiologie [11]. Es kann auch hilfreich sein, sich der eingenommenen Perspektive bewusst zu werden. Das oben schon genannte Problem der Adipositas kann einerseits auf individueller Ebene, andererseits im Kontext einer Familie betrachtet werden, im Kontext von Gemeinden und Settings oder auch als globales Problem: es wären implizit jeweils verschiedene Systemebenen mit Konsequenzen für Interventionen und Evaluationen angesprochen.

Eine in den betroffenen Wissenschaftsbereichen gerne hoch gehaltene Trennung zwischen quantitativen und qualitativen methodischen Verfahren wird angesichts solcher Problemstellungen zu einem Luxus, den sich die Forschungsgemeinschaft zunehmend nicht mehr leisten kann. Notwendig sind Herangehensweisen mit gemischten qualitativen und quantitativen Methoden, eine Herausforderung an auf Evidenz-Synthesen aus gerichtete Forschungskooperationen wie die Cochrane- oder die Campbell-Initiative. Mögliche Erkenntnisse solcher integrierten Evidenzsynthesen (,umbrella reviews“) wären z.B. trans-kontextuelle Adaptionen für eine erfolgversprechende Übertragung von Interventionen auf andere Settings als das ursprünglich gegebene Studiensetting. Für einzelne Fragestellungen wird übrigens berichtet, dass die Anzahl an zusammenfassenden Übersichtsarbeiten inzwischen die der Originalarbeiten übersteigt. Hier ist eine Schieflage zu konstatieren: Grundlage von Reviews, so wichtig die Evidenzsynthesen in einem modernen Wissenschaftsbetrieb sind, bleiben Originalarbeiten.

Solche originale Forschungsarbeiten werden auch in dieser Ausgabe wieder vorgestellt: Zur Auswirkung des demografischen Wandels im Krankenhaus, zu Betriebsstruktur und betrieblichem Gesundheitsmanagement, zu Unterschieden in der Versichertenstruktur von Krankenkassen, zu den gesundheitlichen Folgen von Stalking, zu Sozialstatus und davon abhängigen Risiken, zu den Kosten von Medikamentenreviews, zur Fehlbelegungsprüfung des MDK in der Angiologie und zu Alkohol- und Medikamentenmissbrauch bei pflegebedürftigen älteren Menschen.
In Zusammenhang mit Interventionen in komplexen Systemen wurde die etwas hintergründige Hypothese aufgestellt, dass diese möglicherweise unethisch seien: dann nämlich, wenn sie tatsächlich die Elemente von Unvorhersagbarkeit und chaotischen Ergebnissen in sich trügen (Wolfgang Bödeker). Lässt sich daraus eine Selbstbeschränkung der Forschung auf einfache und überschaubare Interventionen in einem bewusst reduzierten Denkund Handlungsrahmen ableiten? Vielleicht ist auch hier die Schmetterlingsmetapher hilfreich: Der Flug der Schmetterlinge ist nicht von komplexen Absichten getragen, sondern ist eine natürliche und vergleichsweise legitime Anstrengung zur Fortbewegung. Vielleicht gleicht dieses etwas flatterhafte „sich Fortbewegen“ des Schmetterlings in vieler Hinsicht dem wissenschaftlichen Betrieb: gleichsam als Charakteristikum der „Psyche“. Sowenig man Schmetterlingen aus Angst vor Tornados das Flügelschlagen abgewöhnen sollte, sowenig sollte man auch freie geistige Aktivitäten unnötig einengen. Die Metapher des Schmetterlingseffektes hat im Zusammenhang mit Forschungsanstrengungen im Übrigen auch eine positive Seite: nämlich die Hoffnung, dass eine unserer Forschungen, gleich dem Flügelschlag eines Schmetterlings, auch einmal den Wirbelsturm einer unerwarteten, heilsamen und fruchtbaren Erweiterung unseres Denkrahmens bewirken könnte [1,12].

\section{Literatur}

1 Lorenz E. The butterfly effect. In: Lorenz E. The Essence of Chaos. Appendix 1. Seattle: University of Washington Press; 1993; 181-184

2 Lorenz EN. Deterministic nonperiodic flow. J Atmos Sci 1963; 20: 130-141

3 Campbell M, Fitzpatrick R, Haines A et al. Framework for design and evaluation of complex interventions to improve health. BMJ September 16 2000; 321: 694-696

4 Oakley A, Strange V, Bonell C et al. and RIPPLE Study Team. Process evaluation in randomised controlled trials of complex interventions. BMJ 2006; 332: 413-416

5 Swinburn BA, Sacks G, Hall KD et al. The global obesity pandemic: shaped by global drivers and local environments. Lancet 2011; 378: 804-814

6 Rittel H, Webber M. Dilemmas in a general theory of planning. Policy Sciences 1973; 4: 155-169

7 Rothman KJ, Greenland S. Causation and causal inference in epidemiology. Am J Public Health 2005; 95: 144-150

8 Vineis P, Kriebel D. Causal models in epidemiology: past inheritance and genetic future. Environ Health 2006; 5: 21

9 Wildner M. Aristotle and the human genome project. Lancet 2000; 356 (9238): 1360

10 Wildner M. In memory of William of Occam. Lancet 1999; 354 (9196): 2172

11 West GB. The importance of quantitative systemic thinking in medicine. Lancet 2012; 379: 1551-1559

12 Kuhn TS. Die Struktur wissenschaftlicher Revolutionen. Mit einem Postskriptum von 1969. Frankfurt a. M.: Suhrkamp Verlag; 1981 